\title{
Transient infrared thermography testing of Divertor Plasma Facing Components for Nuclear Fusion application
}

\author{
Yashashri Patil, S.S. Khirwadkar, T.Patel, N. Patel, P. Mokaria, P. Patel \\ Institute for Plasma Research, Gandhinagar, Gujarat-382428 \\ ypatil@ipr.res.in
}

\begin{abstract}
Divertor is a plasma facing component of a tokamak such as ITER Tokomak. Divertor is mainly designed to remove the heat flux and particle flux escaping from the core plasma region into Scrape Off Layer of a tokomak. Inner\& Outer vertical targets and Dome are the plasma facing components of ITER Divertor. Tungsten is a most promising plasma facing material joined with the copper alloy heat sink in various geometries. Inner \& Outer vertical targets of the ITER divertor are made of tungsten tiles arranged in monoblock geometry fabricated in straight as well as curved shape. Total 260000 numbers of tungsten monoblocks will be used in the ITER device and having approximately millions of metallurgical joints. During the operation of a fusion reactor, Divertor PFCs have to sustain the steady heat flux of $10-20 \mathrm{MW} / \mathrm{m}^{2}$ resulting in surface temperature in the range $1000^{\circ} \mathrm{C}$ to $2500^{\circ} \mathrm{C}$. Such high heat fluxes are simulated using the High heat flux test facilities with energetic beams of neutral or charged particles such as electrons as a heating source. Infrared imaging system acts as temperature as well as health monitoring tool for Divertor because it covers the wide temperature range starting from room temperature to $3000^{\circ} \mathrm{C}$ with better thermal resolution.
\end{abstract}

Recently a High Heat Flux Test Facility (HHFTF) has been established at IPR to simulate the ITER relevant steady as well as transient heat fluxes on plasma facing components. Straight and curved tungsten monoblock type test mock-ups were fabricated using Hot Radial Pressing (HRP) technique. Two numbers of the straight as well as curved tungsten monoblock type mock-ups were exposed to the high heat flux (HHF) tests up to incident heat flux of $20 \mathrm{MW} / \mathrm{m}^{2}$ using HHFTF at IPR. Transient IR thermography was performed on all the straight as well as curved tungsten monoblock type mock-ups before and after HHF tests using up-graded Transient IR thermography set-up at IPR. Present paper describes results of the Transient IR thermography experiments performed on these test mock-ups.

Keywords: Infrared Thermography, Divertor, HHFTF, Plasma facing components 